\title{
Chimären und Hybride - Ist die Speziesgrenze moralisch relevant?
}

\author{
Marcus Düwell
}

(C) Die Autor(en) 2010. Dieser Artikel ist auf Springerlink.com mit Open Access verfügbar.

Seit einiger Zeit werden zu Forschungszwecken verschiedene Formen von Chimären und Hybriden hergestellt, die die Grenze zwischen Tieren und Menschen überschreiten. Die Beurteilung dieser Forschung führt zu einer Reihe von Regelungsfragen; so wird etwa über die Relevanz dieser Forschung, mögliche Alternativen, Unkenntnis über Forschungsfolgen, Risiken und Unsicherheit, Forschungsfreiheit und vieles mehr gesprochen werden müssen. Doch die Forschung wirft auch die Frage auf, in welcher Hinsicht die Überschreitung und Veränderung der Grenze der menschlichen Spezies grundsätzlich moralisch problematisch ist. Diese Frage wird nicht so einfach zu beantworten sein, auch da man sich wird fragen müssen, wovon man hier überhaupt redet: Wenn etwa eine menschliche Eizelle mit Samenzellen einer nicht-menschlichen Spezies befruchtet wird, kann man fragen, um was für eine Entität es sich überhaupt handelt. Bemisst sich die Zugehörigkeit zur menschlichen Spezies an der Herkunft des biologischen Materials, das zur Befruchtung verwendet wird? Oder geht es um bestimmte Eigenschaften des dadurch erzeugten Wesens? Diese Frage ist fundamental für alle moralischen Bewertungen und rechtlichen Regelungen.

Darüber hinaus ist allerdings unklar, ob wir mit dem Begriff ,,menschlich“ denn überhaupt auf biologische Eigenschaften abheben. Der Begriff „menschlich“ ist sowohl ein Begriff „,biologischer Klassifizierung“ als auch ein „Wertbegriff“; und genau damit stehen wir nun vor einer Reihe von Schwierigkeiten. Wenn von „Menschlichkeit“, „Mitmenschlichkeit“ oder „,der Menschheit in Deiner Person“ geredet wird, dann geht es um einen moralischen Begriff oder zumindest um einen Wertbegriff im Allgemeinen. Nun ist die Zuordnung dieses Wertbegriffs zu bestimmten biologischen Entitäten fragil und problematisch geworden, und das ist eine der zentralen Herausforderungen der Bioethik. Der Hinweis auf den Unterschied zwischen beiden Begriffen ist nun Anlass zu zweifeln, ob die Spezieszugehörigkeit moralisch denn überhaupt relevant ist. Der naheliegende Einwand lautet: Die Berufung auf die Speziesgrenze ist nicht mehr als ein speziesistisches Vorurteil, wonach fälschlicherweise aus der Zugehörigkeit zu einer Gattung auf einen besonderen moralischen

M. Düwell $(\triangle)$

Ethics Institute, Heidelberglaan 8, 3584 CS Utrecht, Niederlande

E-Mail: m.duwell@uu.nl 
Status geschlossen wird. Diese durch Peter Singer populär gewordene Position wird häufig herangezogen, um jede moralische Berufung auf die Speziesgrenze ins Abseits zu befördern $[1,4]$. In ähnlicher Weise kann man auch darauf verweisen, dass in der klassischen Begründung des Status der Menschenwürde bei Kant zwar von der „Menschheit“ die Rede ist, die als Zweck an sich selbst zu behandeln sei, dass dort mit „Menschheit“ aber eindeutig nicht die biologische Spezies bezeichnet ist; vielmehr stellt die rationale Natur des Menschen den Grund der Schutzwürdigkeit dar. Auch bei Kant finden wir also keinen Grund für eine direkte moralische Hochschätzung der Spezies als biologischer Einheit. Darüber hinaus werden nun jedoch Forderungen erhoben, die Begrenzungen, die der menschlichen Spezies anhaften, systematisch zu überwinden und in einer Weise in die Evolution einzugreifen, dass die Spezies, wie wir sie kennen, eines Tages Geschichte sein wird [3].

Die Begründung einer moralischen Verpflichtung zur systematischen Verbesserung der biologischen Ausstattung der menschlichen Gattung hätte jedoch einige Argumentationslasten zu erbringen: So steht man etwa vor dem Problem, dass die entsprechenden biologischen Prozesse nur begrenzt vorhersehbar und beherrschbar sind und dass es verschiedene Vorstellungen darüber gibt, was denn eine wünschenswerte Entwicklung der Menschheit ist. Die Liste der Fragen ist damit kaum erschöpft und ich sehe bislang nicht, dass eine moralische Verpflichtung zur Verbesserung der Evolution überzeugend begründet wäre. Aber grundlegender ist in diesem Kontext die Frage, ob nicht jede mögliche Behauptung einer Schutzwürdigkeit der menschlichen Spezies daran scheitert, dass die biologische Spezies selbst nicht schutzwürdig ist, sondern lediglich Wesen mit Eigenschaften, deren Vorhandensein nicht mit der Speziesgrenze zusammenfallen. Dann würde die moralische Sonderbehandlung der menschlichen Spezies einem speziesistischen Vorurteil entstammen und wäre moralisch sogar zu verurteilen, womit sich dann Begriffe wie „Menschenrechte“ und „Menschenwürde“" moralisch allein schon durch den Speziesbezug verdächtig machen.

Eine grundlegende Zurückweisung eines Schutzanspruchs im Hinblick auf die Spezies ist bislang aber argumentativ keineswegs so überzeugend dargelegt wie häufig angenommen. Die Kritik am Speziesismus hat argumentativ nur überzeugend zeigen können, dass die Zugehörigkeit zu einer besonderen biologischen Spezies als solche keinen Grund darstellt, um die Angehörigen einer solchen Spezies moralisch besonders zu behandeln. Dies Argument ist in jeder Hinsicht triftig. Aber daraus folgt nur, dass die Spezieszugehörigkeit nicht der Grund der Sonderbehandlung sein darf. Weiter folgt daraus nichts. Der moralische Status des Menschen wird im Allgemeinen aus seinen Personeneigenschaften, seiner Handlungs- oder Moralfähigkeit begründet. Die Ausweitung dieses Schutzes (zumindest in abgestufter Hinsicht) auf alle Mitglieder der Gattung kann man kritisieren. Möglicherweise sind einige oder viele der entsprechenden Argumente in der Tat schlecht begründet oder defizitär, aber sie sind auf jeden Fall nicht speziesistisch. Denn die biologische Tatsache der Zugehörigkeit zur Gattung ist dann nicht der Grund, weshalb die Gattung besonders behandelt werden soll. Es würde die Diskussion versachlichen, wenn die Speziesismus-Kritik darauf beschränkt würde, was sie argumentativ leisten kann. Ebenso wenig ist allerdings bislang der Begriff einer Gattungswürde philosophisch profiliert [2]. Es wäre erst zu erweisen, wie die individuelle Menschenwürde systematisch mit der „Würde“ der biologischen Gattung zusammenhängt und wie dies zu Schutzforderungen führen kann. Habermas selbst wird Schwierigkeiten haben, dies argumentativ zu entwickeln, insofern er sich nie darum bemüht hat, der Menschenwürde einen deutlichen systematischen Stellenwert in seiner Philosophie zu geben.

Man kann daher davon ausgehen, dass eine systematische Reflexion auf die moralische Bedeutung der biologischen Spezies eine bislang offene Frage und eine Aufgabe für die bioethische Reflexion darstellt. Für diese Diskussion wird sich wesentlich die Frage stellen, 
welche moralische Bedeutung der kontingenten biologischen Erscheinungsform des Menschen zukommt. Wenn es zutrifft, dass dem Menschen ein besonderer moralischer Schutz nur zukommt, weil er personale Eigenschaften hat, so sehen wir uns mit dem Problem konfrontiert, dass man personale Eigenschaften nicht so identifizieren kann, wie man Körpergröße oder Haarfarbe deskriptiv erfassen kann. Es kann sogar philosophisch gefragt werden, ob sich uns die Personalität nicht aus grundsätzlichen Gründen phänomenal unzugänglich bleiben muss. Daraus folgt nun nicht, dass Personalität moralphilosophisch unwichtig wird. Es folgt aber, dass es moralphilosophisch wesentliche Gründe dafür geben kann, warum der moralische Schutz an der phänomenalen Erscheinung des Menschen und damit auch der kontingenten, biologischen Gestalt des Menschen festgemacht wird. Um es an einem einfachen Beispiel zu verdeutlichen: Wenn es darum geht, ob einem Wesen Menschenrechte zukommen, gestatten wir es keiner Behörde festzulegen, ob es sich jetzt bei diesem Wesen um eine Person handelt, sondern wir gehen von der Gattungszugehörigkeit als Bezugsgröße aus. Wenn es darum geht, einen effektiven Schutz von Personen im rechtlich-politischen Raum zu etablieren, so gibt es dazu auch kaum eine Alternative. Ebenso ist es schwer vorstellbar, dass wir in der Lage sind, Personen mit Respekt zu behandeln, wenn wir nicht die phänomenale Erscheinungsform des Menschen als Bezugsgröße hätten. Wenn wir uns stets fragen müssten, ob das Wesen, das uns begegnet, ein Wesen ist, das Respekt verdient, wäre es schwer vorstellbar, dass der Respekt vor dem anderen zu einem lebensweltlich etablierten Verhaltensstandard wird, den wir voneinander erwarten und der geteilte Sozialstrukturen prägt. Insofern ist die kontingente biologische Erscheinung des Menschen, wie wir sie kennen, bis dato von wesentlicher Bedeutung für die Realisierung des moralischen Schutzes, den eine Ethik des Respekts vor der Person des Anderen uns abverlangt.

Damit ist nicht gesagt, dass nicht eine Gesellschaft vorstellbar wäre, in der dies anders ist; als Star-Trek-Fan habe ich Phantasie genug, um mir Alternativen vorzustellen. Auch kann durch die Betonung einer prinzipiellen moralischen Bedeutung der biologischen Speziesgrenze allein nicht über die Zulässigkeit einzelner Laborexperimente mit Chimären und Hybriden entschieden werden. Und schon gar nicht folgt aus diesen Überlegungen, dass Wesen, die dieser Spezies nicht angehören, moralisch nicht schützenswert seien. Aber es wird doch deutlich, dass die Frage nach der moralischen Bedeutung der biologischen Spezies eine theoretische Herausforderung für die Bioethik ist, die es systematisch aufzuarbeiten gilt. Bislang wurden die politischen Kontroversen um den moralischen und rechtlichen Schutz von Menschen, von denen wir nicht vermuten, dass sie aktuell über Personeneigenschaften verfügen, mit politischen Kompromissen beantwortet. Damit blieb der moralische Status der menschlichen Gattung moralisch und rechtlich uneindeutig und systematisch wenig transparent. Die Diskussion um Chimären und Hybride wird die Unübersichtlichkeit wahrscheinlich erhöhen. Die passende Antwort der Bioethik schiene mir eine systematische Diskussion über das Verhältnis von moralischem Status, moralischen Schutzgütern und biologischer Spezies. Wir sollten versuchen, genauer zu verstehen, warum wir welchen biologischen Entitäten welchen moralischen Schutz zukommen lassen. Schnelle Einigungen und Kompromisse sind auf diesem Feld nicht zu erwarten. Doch ein systematischeres Verständnis könnte das Vertrauen in die biopolitische Gesetzgebung erhöhen, deren Strategien der Kompromissbildung sich vom Verständnis des Bürgers bereits deutlich entfernt haben. Und die Glaubwürdigkeit der Bioethik würde durch eine solche Diskussion sicherlich auch erhöht. ${ }^{1}$

\footnotetext{
${ }^{1}$ Dieser Text basiert auf Überlegungen im Zusammenhang mit dem Europäischen Projekt Chimbrids, bei dem der Verfasser die Federführung für den Ethik-Teil hatte (vgl. [5], S. 61-79, 571-592).
} 
Open Access Dieser Artikel unterliegt den Bedingungen der Creative Commons Attribution Noncommercial License. Dadurch sind die nichtkommerzielle Nutzung, Verteilung und Reproduktion erlaubt, sofern der/die Originalautor/en und die Quelle angegeben sind.

\section{Literatur}

1. Düwell M (2009) Philosophical pressupositions of practical ethics. In: Schaler J (Hrsg) Singer under fire. The dangerous ethicist faces his critics. Open Court, Chicago, S 395-419

2. Habermas J (2001) Die Zukunft der menschlichen Natur. Auf dem Weg zu einer liberalen Eugenik? Suhrkamp, Frankfurt a.M.

3. Harris J (2007) Enhancing evolution. The ethical case of making people better. Princeton University Press, Princeton

4. Singer P (1994) Praktische Ethik. Neuausgabe. Reclam, Stuttgart

5. Taupitz J, Weschka M (Hrsg) (2009) CHIMBRIDS - chimeras and hybrids in comparative European and international research: scientific, ethical, philosophical and legal aspects. Springer, Berlin 